\title{
Imaging of charge trapping in distorted carbon nanotubes by $x$-ray excited scanning probe microscopy
}

\author{
Masashi Ishii, ${ }^{1, a)}$ Bruce Hamilton, ${ }^{2}$ and Nigel Poolton ${ }^{3}$ \\ ${ }^{1}$ National Institute for Materials Science (NIMS), 1-2-1 Sengen, Tsukuba, Ibaraki 305-0047, Japan \\ ${ }^{2}$ Photon Science Institute, The University of Manchester, Manchester M13 9PL, United Kingdom \\ ${ }^{3}$ Delft University of Technology, Mekelweg 15, 2629 JB Delft, The Netherlands
}

(Received 10 August 2008; accepted 9 October 2008; published online 25 November 2008)

\begin{abstract}
We have observed the distribution of electron trapping centers on distorted carbon nanotubes (CNTs) by a unique $\mathrm{x}$-ray analysis technique that has both elemental and spatial selectivities. This technique involves the use of scanning probe microscopy (SPM) under synchrotron radiation excitation of the inner shell of carbon. The probe detects the Coulomb force that results from the relaxation of an electron bound to a defect site into the core hole state created by x-ray photon absorption. This results in a change in charge state of the defect. At the spatial resolution provided by the SPM technique, we observed the electron trapping centers distributed on the compressed and torsionally distorted CNTs. This direct association of mechanical distortion with defect sites indicates a potential risk of deterioration of electric properties during the fabrication and processing of CNT networks. @ 2008 American Institute of Physics. [DOI: 10.1063/1.3029725]
\end{abstract}

\section{INTRODUCTION}

A carbon nanotube (CNT) can act as an ideal electron channel, ${ }^{1}$ and well-designed CNT networks can be used to realize integrated circuits. ${ }^{2}$ In such networks, however, unexpected distortions of CNTs at basic subassemblies such as contacts and branches occasionally occur during fabrication. The local distortions modulate the electronic states of $\mathrm{CNTs}^{3-5}$ resulting in the discontinuity or degradation of electron transport. Knowledge about the origin and cause of electronic modulation in CNT networks is crucial to realize integrated circuits; however, undesirable electronic modulations cannot be easily detected in the images of CNTs obtained by simple microscopic techniques. ${ }^{6}$ In an attempt to obtain such information, we applied a unique electronic-state mapping technique ${ }^{7}$ developed by our group to CNT networks. In this technique, we introduce $\mathrm{x}$ rays as an excitation source for the structure accessed by the scanning probe microscope (SPM) tip; this has several advantages. First, this technique provides chemical selectivity by virtue of resonant $\mathrm{x}$-ray excitation of inner-shell electrons, i.e., of $\mathrm{C}$ atoms in the present case. This makes it possible to discriminate electronic states intrinsic to the CNT in a background, such states which may be associated with a substrate. Second, the spatial resolution achieved in this technique is superior to that of conventional $\mathrm{x}$-ray analysis techniques. This is because the probed area is restricted to a small point under the scanning tip; therefore, the distribution of the electronic states can be spatially resolved using the nanoscale near field attributes of the SPM.

Details of SPM combined with x-ray excitation have been described in previous papers. ${ }^{7,8}$ Unlike other related works, ${ }^{9-14}$ the SPM used in this study was configured for electrostatic force microscopy (EFM), ${ }^{15-18}$ and the x-ray photons were produced by synchrotron radiation (SR). We

${ }^{a)}$ Electronic mail: ishii.masashi@nims.go.jp. denote EFM combined with the X-ray source as X-EFM. In a previous study, we applied X-EFM to the observation of surface defects on ultrathin $\mathrm{SiO}_{2}$ films and realized a spatial resolution of less than $1 \mathrm{~nm}$ in chemical state mapping. ${ }^{7}$ A key aspect of detecting the $\mathrm{x}$-ray matter interactions with the EFM tip concerns the detail of electron transfer between an electron occupied defect state and a core hole produced by $\mathrm{x}$-ray absorption at a parent atom at the defect site. Near the $\mathrm{x}$-ray pumped sample surface, inner-shell excitation creates a hot core hole, which rapidly thermalizes and (on a slower time scale) will be filled by an electron; in the absence of a nearby bound electron, the core hole will be filled by a valence electron. However if the $\mathrm{x}$-ray excited atom forms part of a defect complex, there will be some probability that the core hole is directly filled by the electron occupying the equilibrium bound state. For example, the bound electron may form a dangling bond associated directly with the parent atom and, consequently, a substantial wave function overlap may exist between the electron and a relaxed core hole, making this direct transfer from bound electron to core hole state favorable. In this case, the core holes produced at the innershell excitation contribute directly to defect charging at distorted regions of the CNT, which are rich in electron trapping centers; the EFM allows us to image such localized charging events on the nanoscale. The fundamental charging process is equivalent to the photoionization of the distorted regions of the CNT. When the photoionized state has a relatively long lifetime, say of the order of milliseconds, it can be mere readily averaged and imaged EFM. On the other hand, the other possible photocharged sites, such as the capacitance built into a CNT/insulator/substrate structure, are immediately relaxed by free charges, resulting in no X-EFM image.

\section{EXPERIMENTS}

The experiments were carried out using beamline multipole wiggler (MPW) 6.1 of Synchrotron Radiation Source 



FIG. 1. Experimental setup of X-EFM system and method for detection of $\mathrm{x}$-ray photoionization in this system (inset).

(SRS) at Daresbury Laboratory, United Kingdom. ${ }^{19}$ This beamline provides tunable soft $\mathrm{x}$ rays from the MPW insertion device. In our study, the $\mathrm{x}$-ray photon energy was tuned around the $K$-edge energy of carbon, i.e., $284 \mathrm{eV}$, with a monochromator in order to verify the effects of the selective inner-shell excitation of $\mathrm{C}$ atoms. The $\mathrm{x}$ rays were focused on the sample surface under the EFM probe, and their beam size at the X-EFM probe tip was approximately $1 \mathrm{~mm}^{2}$.

Figure 1 shows the setup of the X-EFM system used in the imaging experiments. The probe head of the X-EFM system was custom designed for SR beam irradiation, and the system was manufactured by Unisoku Co., Ltd. X-EFM images were obtained with a noncontact probe oscillated at an eigenfrequency $f_{e}$ of the cantilever, utilizing a feedback loop between a piezoelectric sensor and an actuator. ${ }^{20,21}$ For electrostatic force detection, the probe was coated with conducting metals; the thickness of the coated layers was approximately $5 \mathrm{~nm}$ for chromium and $20 \mathrm{~nm}$ for gold. This allows for the application of both ac and dc biases between tip and sample, which are required for Coulomb force detection. In order to distinguish the electrostatic force from other forces on the cantilever, e.g., van der Walls force, we employed a lock-in amplification of force signals associated with ac bias voltage oscillator. This is shown schematically in the inset of Fig. 1; the probe is grounded and the voltage oscillator produces an electric field between the tip of the probe and the sample. The cantilever responds to the electrostatic force gradient that is modulated by the varying electric field strength produced by the ac bias at frequency $f_{v}$. The force gradient produced by the Coulomb interaction of the purely capacitive (displacement) charges on tip and sample produces an amplitude modulation of $2 f_{v}$ on the cantilever mo-

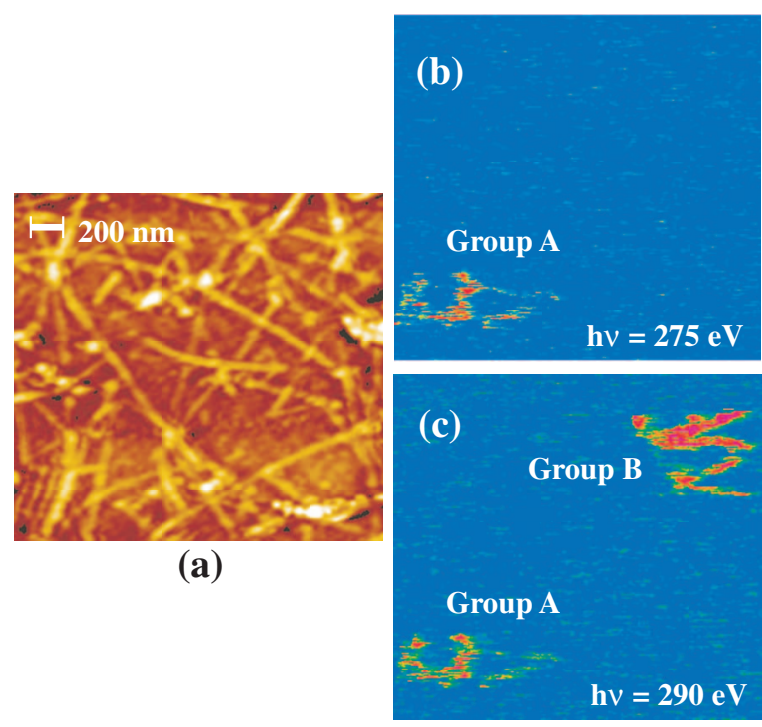

FIG. 2. (Color) (a) AFM image of CNTs on $\mathrm{SiO}_{2} / \mathrm{Si}$ substrate and X-EFM images at (b) $h \nu=275 \mathrm{eV}$ and (c) $h \nu=290 \mathrm{eV}$.

tion. Any fixed charge near the surface will produce a Coulomb interaction with the tip capacitive charge, which leads to a $f_{v}$ modulation of the cantilever oscillation, and this was the component sensed in this work. When $\mathrm{x}$ rays interact with the sample, photoionization changes the charge state of the distorted CNTs, as described in Sec. I. The charge state causes a change in $\Delta F$ in the electrostatic force, which can be obtained by deconvolution of the oscillated signal. In our experiments, the frequency $f_{v}$ of the ac oscillator was set to 3-10 kHz lower than $f_{e} \sim 110 \mathrm{kHz}$. The X-EFM signal was detected with a low-pass filter (NF Electronic Instruments, model 3314) with a cutoff frequency $f_{c}$ of $50 \mathrm{kHz}$. It is important to note that by setting $f_{c}$ such that $f_{v}<f_{c}<f_{e}$, selective detection of the electrostatic force can be achieved. A topographic image and a photoionization image are simultaneously obtained by atomic force microscopy (AFM) using $f_{e}$ and by X-EFM using $f_{v}$, respectively, without interference.

The sample consisted of randomly distributed singlewalled CNTs grown on a thin $\mathrm{SiO}_{2}$ film by a vapor phase method using an $\mathrm{Fe}$ nanoparticle catalyst. The heavily doped $n$-type Si substrate served as an electrode for electrostatic force detection. The CNTs were electrically isolated due to the presence of the approximately 50 -nm-thick $\mathrm{SiO}_{2}$ film, which enables the photoionized CNTs to attain a metastable state with a relatively long lifetime. ${ }^{7}$ Although the CNT network is not identical to the network of electric circuits, condensed CNTs provide a practically similar situation to artificial structures.

\section{RESULTS AND DISCUSSION}

\section{A. Electron trapping centers in compressed CNTs}

Figure 2(a) shows a noncontact AFM topography image of the sample surface, which indicates that the CNTs and their bundles are randomly distributed on the surface. Some of them cross each other and some bundles branch into thinner wires at the center. At these crosses and branches, the CNTs undergo distortion due to compression, stretching, tor- 


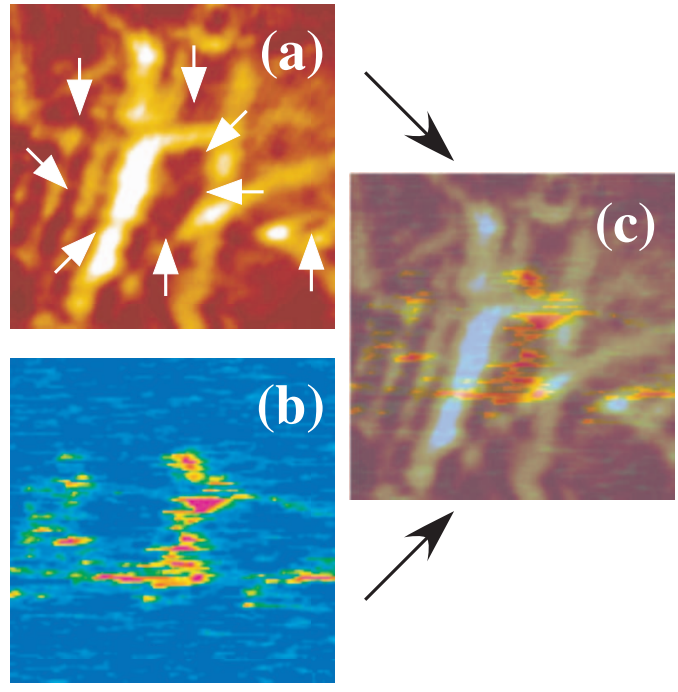

FIG. 3. (Color) (a) AFM and (b) X-EFM images of group A. These images are overlapped in a single frame in (c).

sion, etc. ${ }^{6}$ Considering the flexibility of CNTs, not all distortions would be expected to form the trapping states; therefore, although the topographic AFM image shows all distortions, it cannot specifically indicate the trapping centers.

Figures 2(b) and 2(c) show X-EFM images of the same surface area as those shown in the topographic AFM image [Fig. 2(a)]. The x-ray photon energy $(h \nu)$ was $275 \mathrm{eV}$ for obtaining the image shown in Fig. 2(b) and $290 \mathrm{eV}$ for that shown in Fig. 2(c). These photon energies correspond to those below and above the $K$-edge of carbon $(284 \mathrm{eV})$; only the photons with $h \nu=290 \mathrm{eV}$, i.e., energy greater than the $K$-edge energy, excite the inner-shell electrons of the $\mathrm{C}$ atoms. Selective inner-shell excitation of $\mathrm{C}$ atoms is thus achieved by controlling $h \nu$ and enables us to distinguish the electron trapping centers in CNTs from those in the $\mathrm{SiO}_{2}$ thin film.

From the X-EFM images shown in Figs. 2(b) and 2(c), it is found that the sample has two groups of electron trapping centers, labeled group A and group B. The trapping centers are not uniformly distributed over the sample but are localized at specific sites. It should be noted that group A could be imaged independent of $h \nu$, while group B produces switchable X-EFM signal, turned only by photon energy, $h \nu$ at the $\mathrm{C} K$ edge. This switchable signal proves that group $\mathrm{B}$ is related to the $\mathrm{C}$ atoms, indicating the element selectivity of the $\mathrm{X}$-EFM technique.

For more detailed understanding of element selectivity, magnified AFM and X-EFM images of group A trapping centers (independent of $h \nu$ ) are shown in Figs. 3(a) and 3(b), respectively. Figure 3(c) shows the two images in Figs. 3(a) and 3(b) overlapped in a single frame to identify the origin of the X-FEM signal. Typical positions at which intense $\mathrm{X}$-EFM signals were observed are indicated by arrows in the AFM image. The arrows indicate a bare $\mathrm{SiO}_{2}$ area rather than the CNT occupied area, indicating that group A is related to a surface defect of $\mathrm{SiO}_{2}{ }^{18}$ In particular, the intense X-EFM signal at the specified $\mathrm{SiO}_{2}$ area suggests a concentration of higher density or deeper level defects than that is present in

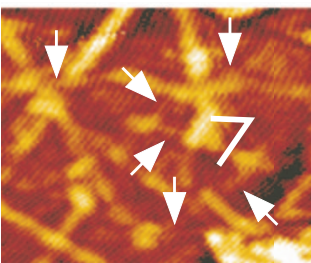

(a)

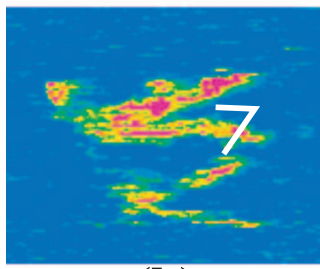

(b)

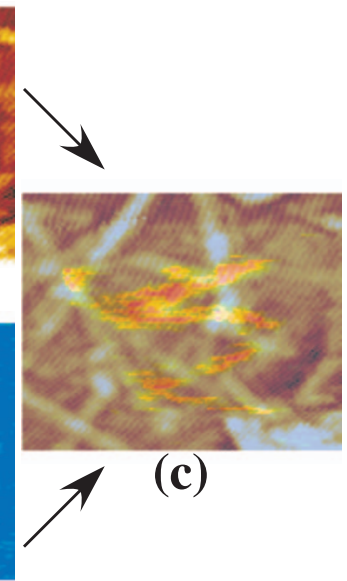

FIG. 4. (Color) (a) AFM and (b) X-EFM images of group B. These images are overlapped in a frame in (c).

the other surface area. Since the $\mathrm{SiO}_{2}$ defects are independent of the $\mathrm{C}$ atoms, the X-EFM image of group A should be insensitive to the slight change in $h \nu$ around the $\mathrm{C} K$-edge. This consideration is consistent with the experimental results shown in Fig. 2.

On the other hand, group B with the switchable X-EFM signal (Fig. 2) belongs to a specific CNT. Magnified AFM and X-EFM images of group B are shown in Figs. 4(a) and 4(b), respectively. Figure 4(c) shows the two images in Figs. 4(a) and 4(b) overlapped in a single frame to identify the origin of the X-FEM signal. Typical positions of intense $\mathrm{X}$-EFM signals are represented by arrows in the AFM image. As described below, we found a common feature in the CNTs indicated by these arrows. Figure 5(a) shows the AFM profile along the white line indicated in Fig. 4(a). The AFM profile consists of a double peak, indicating that the line crosses over two CNTs: one located at a higher position (left peak) and the other at a lower position (right peak), as shown in the inset of Fig. 5(a). We also show corresponding X-EFM

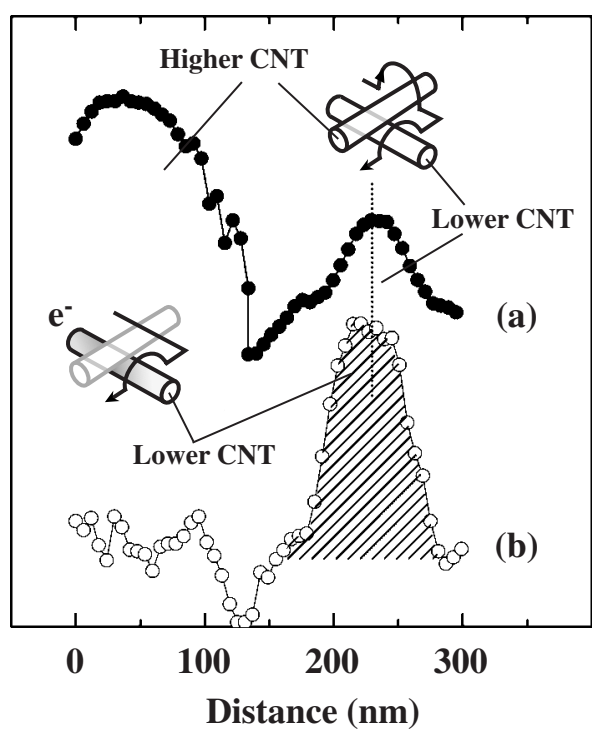

FIG. 5. (a) AFM and (b) X-EFM profile along a white line indicated in Fig. 4(a). 


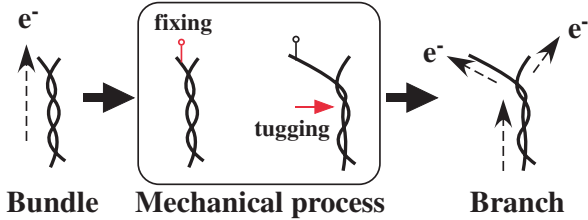

(a)

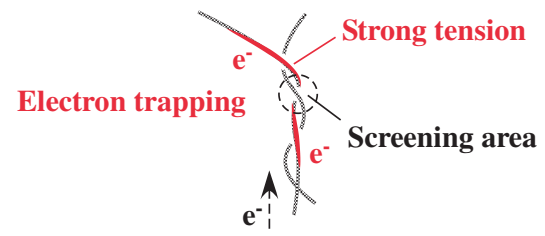

(b)

FIG. 6. (Color) (a) Mechanical process for CNT branch formation and (b) probable distribution of electron trapping centers produced by the process.

profile along the same line in Fig. 5(b). In contrast to the AFM image, the X-EFM profile consists of a single peak that appears at the lower CNT position. This result reveals that electron trapping centers were concentrated on the lower CNT. Interestingly, all group B trapping centers were found to be located on the lower CNTs at crosses. This finding can be explained as follows. The upper CNTs were flexible enough to recover their original shape after some amount of temporary compression, while the lower CNTs were irreversibly deformed. The permanent distortion of the lower CNTs, which probably led to a defect, modified the electronic states, resulting in the concentration of the electron trapping centers on the lower CNTs. From this partial process, it is concluded that the lower CNTs receive additional pressure due to the upper CNTs, and/or the upper CNTs prevent the recovery of the original shape of the lower CNTs after deformation.

Thus, by using the X-EFM technique, we can specifically identify the distorted CNTs that have electron trapping centers in their networks. Continuous photon energy scan using a static probe on the distorted CNTs is expected to provide $\mathrm{x}$-ray absorption spectrum intrinsic to the structure. For this purpose, we are trying to solve problems of thermal drift of the probe and charge refilling to photoionized CNTs. Although the detailed electronic structure has never been clarified, it is notable that the X-EFM at $290 \mathrm{eV}$ provides a specific image of $\pi^{*}$ state excitation. ${ }^{22-24}$ The $\pi^{*}$ state is sensitive to chemical modification of CNTs and is probably changed by the distortions found in this study.

\section{B. Electron trapping centers in torsional CNTs}

In this section, we present another example of the imaging distorted CNTs with associated electron trapping centers, viz., electronic degradation observed at a branch of a CNT bundle. As shown in Fig. 6(a), bundles were formed from at least two torsional CNTs. ${ }^{25}$ The branches of CNTs were possibly formed by a mechanical process such as fixing of one CNT and "tugging" of another. The resulting branches are expected to function as electron splitters in CNT networks. However, as shown in Fig. 6(b), the mechanical process in-

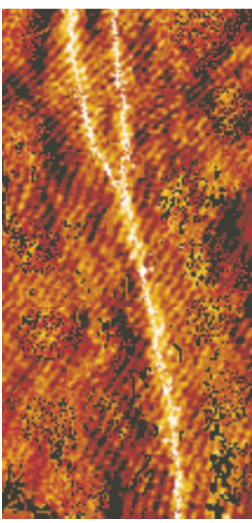

(a)

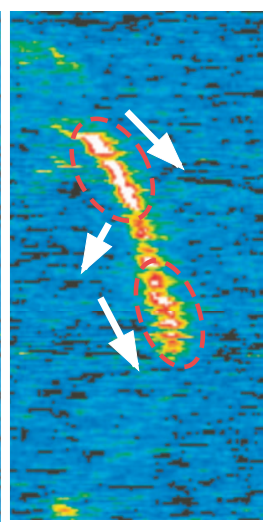

(b)

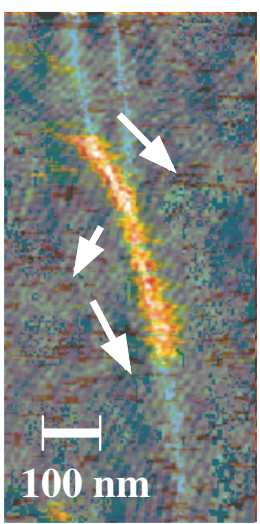

(c)
FIG. 7. (Color) (a) AFM and (b) X-EFM images of CNT branch. These images are overlapped in a frame in (c).

duced a strong tension on the "tugged" CNT and deformed it, resulting in the formation of electron trapping centers at the branch.

Figure 7 shows AFM [Fig. 7(a)] and X-EFM [Fig. 7(b)] images of a CNT branch. The X-EFM image was obtained at $h \nu=290 \mathrm{eV}$; the $\mathrm{C}$ atoms could be excited by the $\mathrm{x}$ rays. The $\mathrm{X}$-EFM image reveals the distribution of electron trapping centers, while the AFM image shows the topography of the branch. On the basis of the AFM and X-EFM images overlapped in a frame [Fig. 7(c)], it is found that the electron trapping centers are distributed on one of the torsional CNTs. It should be noted that the intense X-EFM signal is divided into two parts, as indicated by the dashed circles, suggesting that the distorted CNT is moved behind at the center and the electron trapping centers are screened by another CNT [see Fig. 6(b)]. In other words, the electron trapping centers are confined within one and one-half turn of the torsional CNTs around the branch.

\section{CONCLUSION}

We imaged electron trapping centers in a CNT network by using EFM excited with a synchrotron $\mathrm{X}$-ray source $(\mathrm{X}$ EFM). We have shown that such X-EFM can emphasize the electron trapping centers intrinsic to CNTs due to the selective inner-shell excitation of $\mathrm{C}$ atoms. Moreover, X-EFM offers a high spatial resolution of nanometer scale, and X-ray photoionization is detected with the near field resolution of the scanning probe. The trapping centers are found on compressed CNTs at points where two CNTs cross each other and at the branches on a torsional CNT. This finding indicates that an external force that deforms the CNTs degrades their electric properties for specific distortional events. Crosses and branches of CNTs are indispensable geometries in a subassembly of CNT networks and are necessary to fabricate functional circuits. The experimental results of $\mathrm{X}$-EFM reported here indicate that the effects of mechanical processes require careful control and study in order to optimize the fabrication of CNT circuits.

${ }^{1}$ B. Chen, H. Lin, T. Huang, J. Wei, H. Wang, M. Tsai, T. Chao, and R. Sordan, Appl. Phys. Lett. 88, 093502 (2006). 
${ }^{2}$ R.Sordan, K. Balasubramanian, M. Burghard, and K. Kern, Appl. Phys Lett. 88, 053119 (2006).

${ }^{3}$ F. Bussolotti, L. D’Ortenzi, V. Grossi, L. Lozzi, S. Santucci, and M. Passacantand, Phys. Rev. B 76, 125415 (2007).

${ }^{4}$ S. Agrawal, M. S. Raghuveer, H. Li, and G. Ramanath, Appl. Phys. Lett. 90, 193104 (2007).

${ }^{5}$ P. Castrucci, M. Scarselli, M. De Crescenzi, M. A. El Khakani, F. Rosei, N. Braidy, and J.-H. Yi, Appl. Phys. Lett. 85, 3857 (2004).

${ }^{6}$ P. Castrucci, M. Scarselli, M. De Crescenzi, M. Diociaiuti, P. Chistolini, M. A. El Khakania, and F. Rosei, Appl. Phys. Lett. 87, 103106 (2005).

${ }^{7}$ M. Ishii, B. Hamilton, N. R. J. Poolton, N. Rigopoulos, S. De Gendt, and K. Sakurai, Appl. Phys. Lett. 90, 063101 (2007).

${ }^{8}$ M. Ishii, N. Rigopoulos, N. Poolton, and B. Hamilton, Physica B 376-377, 950 (2006).

${ }^{9}$ K. Tsuji, K. Wagatsuma, K. Sugiyama, K. Hiraga, and Y. Waseda, Surf. Interface Anal. 27, 132 (1999).

${ }^{10}$ S. M. Gray, J. Electron Spectrosc. Relat. Phenom. 109, 183 (2000)

${ }^{11}$ T. Matsushima, T. Okuda, T. Eguchi, M. Ono, A. Harasawa, T. Wakita, A. Kataoka, M. Hamada, A. Kamoshida, Y. Hasegawa, and T. Kinoshita, Rev. Sci. Instrum. 75, 2149 (2004).

${ }^{12}$ A. Saito, J. Maruyama, K. Manabe, K. Kitamoto, K. Takahashi, K. Takami, S. Hirotsune, Y. Takagi, Y. Tanaka, D. Miwa, M. Yabashi, M. Ishii, M. A. Kasaya, S. Shin, T. Ishikawa, Y. Kuwahara, and M. Aono, Jpn. J. Appl. Phys., Part 1 45, 1913 (2006).

${ }^{13}$ S. Suzuki, Y. Koike, K. Fujikawa, W. J. Chun, M. Nomura, and K.
Asakura, Chem. Lett. 33, 636 (2004).

${ }^{14}$ S. Larcheri, F. Roccaa, F. Jandard, D. Pailharey, R. Graziola, A. Kuzmin, and J. Purans, Rev. Sci. Instrum. 79, 013702 (2008).

${ }^{15}$ O. Vatel and M. Tanimoto, J. Appl. Phys. 77, 2358 (1995).

${ }^{16}$ K. P. Puntambekar, P. V. Pesavento, and C. D. Frisbie, Appl. Phys. Lett. 83, 5539 (2003).

${ }^{17}$ C. Ng, T. Chen, H. Lau, Y. Liu, M. Tse, O. Tan, and V. Lim, Appl. Phys. Lett. 85, 2941 (2004).

${ }^{18}$ M. Ishii and B. Hamilton, Appl. Phys. Lett. 85, 1610 (2004).

${ }^{19}$ M. Bowler, J. B. West, F. M. Quinn, D. M. P. Holland, B. Fell, P. A. Hatherly, I. Humphrey, W. R. Flavell, and B. Hamilton, Surf. Rev. Lett. 9, 577 (2002).

${ }^{20}$ N. Satoh, K. Kobayashi, S. Watanabe, T. Fujii, T. Horiuchi, H. Yamada, and K. Matsushige, Jpn. J. Appl. Phys.,Part 1 42, 4878 (2003).

${ }^{21}$ N. Satoh, K. Kobayashi, S. Watanabe, T. Fujii, T. Horiuchi, H. Yamada, and K. Matsushige, Appl. Surf. Sci. 188, 425 (2002).

${ }^{22}$ C. L. Yueh, J. C. Jan, J. W. Chiou, W. F. Pong, M.-H. Tsai, Y. K. Chang, Y. Y. Chen, Y. F. Lee, P. K. Tseng, S. L. Wei, C. Y. Wen, L. C. Chen, and K. H. Chen, Appl. Phys. Lett. 79, 3179 (2001).

${ }^{23}$ Z. Li, L. Zhang, D. E. Resasco, B. S. Mun, and F. G. Requejo, Appl. Phys. Lett. 90, 103115 (2007).

${ }^{24}$ S. C. Ray, C. W. Pao, H. M. Tsai, J. W. Chiou, W. F. Ponga, C. W. Chen, M.-H. Tsai, P. Papakonstantinou, L. C. Chen, K. H. Chen, and W. G. Graham, Appl. Phys. Lett. 90, 192107 (2007).

${ }^{25}$ K. M. Liew, C. H. Wong, and M. J. Tan, J. Appl. Phys. 99, 114312 (2006). 